\title{
Apuntes para la elaboración de una historia de la medicina tradicional andina*
}

\section{Notes for the development of a history of traditional Andean medicine}

Erick Devoto Bazán**

Universidad de Lima

\section{Resumen}

En la actualidad, la medicina occidental ve con mayor interés las prácticas curativas tradicionales por ser estas menos invasivas y por contar con recetas y tratamientos de comprobada eficacia. Este acercamiento se ha logrado gracias a la ayuda de estudios históricos y antropológicos. Sin embargo, en el caso de la medicina tradicional peruana, la historiografía no ha trabajado con profundidad el tema. Este artículo pretende alentar el desarrollo de nuevos trabajos sobre este tópico y para ello reseña brevemente las fuentes escritas referentes a la medicina andina, desde las recopilaciones de los primeros cronistas en el XVI hasta los estudios actuales.

* Este artículo tiene su origen en el curso de Seminario de historia andina (año 2011), de la maestría en historia de la Universidad Nacional Mayor de San Marcos. Agradezco a mi profesor y amigo Fernando Armas Asín por haberme inspirado a indagar sobre este tema.

** Licenciado en historia por la Pontificia Universidad Católica del Perú (2003). Egresado de la maestría en historia de la Universidad Nacional Mayor de San Marcos (2013).

Contacto: erickdevoto@yahoo.com 
Palabras clave: Perú, Historia, cultura andina, medicina tradicional, siglos XVI- XXI

\begin{abstract}
Currently, growing numbers of Western medical practitioners consider traditional healing practices and treatments as less invasive and more effective. This is due largely to advances in research within the fields of history and anthropology. However, the historiography of Peruvian traditional healing practices has not been fully developed yet. This article examines some of the main contributions on this topic so far and provides a brief overview of written sources on Andean traditional medicine since pre-Hispanic times up to the present.
\end{abstract}

Key words: Peru, History, andean culture, traditional healing practices, XVI-XXI centuries$$
* * *
$$

"El médico no debe tener miedo de ensuciarse los fundillos del pantalón entrando a la casa del curandero y sentándose a su mesa".

Elmer Huerta (2015: A14, citando a su maestro Max Arnillas)
\end{abstract}

Hace pocos meses, el Dr. Elmer Huerta - destacado oncólogo y laureado médico peruano- publicó un artículo en el diario El Comercio de Lima (2015: A14), en el que daba cuenta de un experimento realizado en Gran Bretaña, que demostró la eficacia de una receta de más de mil años para curar las infecciones purulentas de los ojos o conjuntivitis. El remedio medieval, del siglo IX a. C., fue reproducido tal 
como se indicaba en el Leechbook de Bald del rey Alfredo de Inglaterra y demostró tener el poder de eliminar a una superbacteria como el Staphylococcus aureus. Las superbacterias constituyen una de las mayores preocupaciones de la medicina moderna, ya que tienen un alto nivel de resistencia a los antibióticos y crean dudas sobre la buena salud pública del futuro. El éxito de este remedio premoderno inglés lleva al Dr. Huerta a plantear la necesidad de investigar más y mejor las medicinas tradicionales del mundo, no solo para buscar soluciones alternativas a los antibióticos, sino también para fortalecer la inmunología de los seres humanos. Huerta lo propone en el contexto de un mayor interés dentro de la ciencia médica por seguir métodos naturales, menos invasivos. La sabiduría tradicional para Huerta tiene un valor sumamente importante:

[...] qué lástima que nuestros gloriosos antepasados paracas, mochicas, quechuas y aimaras no supieran escribir, ¡cuántas maravillosas y efectivas recetas nos hubieran dejado! ¡Qué maravilloso hubiera podido (sic) tener la receta exacta de los anestésicos que usaban para hacer las trepanaciones craneanas que efectuaban para operar tumores cerebrales o de los preparados y emplastos que usaban para que no se les infecte las heridas!

Y por último, es muy importante que los científicos modernos sepamos valorar y respetar a los empíricos de todos los pueblos del mundo. Su arte y su práctica es de mucho valor $[\ldots]$

Coincidimos con el Dr. Huerta en su lamento por lo que se ha perdido de la medicinal andina tradicional, pero creemos que aún es posible rescatar mucho de ese saber antiguo con herramientas como la historia o la antropología y que, mejor aún, podremos aplicar ese saber en nuestra salud del futuro. En este artículo se hace un breve recuento de la evolución de la medicina tradicional andina desde los tiempos incaicos 
(sobre los que los españoles dejaron los primeros escritos) hasta el presente y se reseñan las principales fuentes que conocemos sobre los médicos andinos y sus prácticas. Se espera que este primer paso ayude a consolidar una importante línea historiográfica que aproveche el saber de los antiguos para tener una mejor salud en el futuro y que también nos permita fortalecer nuestra identidad como nación mestiza, en la que la cultura andina tradicional sea valorada como se merece.

\section{Las primeras fuentes: los documentos hispanoandinos}

Las primeras fuentes sobre la medicina tradicional andina, como ocurre con la historia peruana en general, lo constituyen los documentos coloniales, en particular las crónicas. Esto se debe a que los hombres precolombinos no manejaron la escritura y por ello no dejaron testimonio escrito sobre sus actividades ${ }^{1}$. A partir del arribo hispánico, tanto españoles como indígenas occidentalizados dejaron testimonio de las prácticas e ideas curativas de los antiguos peruanos y entre ellos destaca el padre jesuita Bernabé Cobo.

Quizá a uno de los primeros cronistas que se debe mencionar es al Inca Garcilaso de la Vega (1539-1616), quien en 1609 publicó su clásica obra Comentarios reales de los incas, en la que describe muchas de las curaciones indígenas realizadas a los conquistadores hispanos, tanto en sus luchas contra el

82 incanato como en sus guerras civiles. Por ejemplo, Garcilaso da cuenta de una curación con estiércol de auquénido mezclado con un "sebo" particular, que salvó a muchos heridos de guerra de la tropa encomendera de Gonzalo Pizarro:

1 Es cierto que existen algunas teorías históricas que señalan una posibilidad de lenguaje prehispánico en los quipus, pero esto no ha sido comprobado ni validado del todo. 
Los indios de servicio que los españoles tenian consigo digeron a sus amos: Que se curar con aquel sebo y ellos mismos lo derritieron en dos cascos de hierro que sus amos acertaron á llevar y trujeron del estiercol del ganado de aquella tierra que por aquellos campos había mucho y hecho polvos la mefclaban con el sebo y afi caliente quanto se podia sufrir lo echavan en las heridas y las llenavan por hondas que eftuviefen y con la mismo cvraron sus cavallos y fe confortaron con la merced que Dios les hico de aquel remedio; que fue tal, que sin mas cvra ni otra medicina alguna sanaron [...] (Citado en Valdizán 1911) (El subrayado de aquí en adelante es del autor).

Otra crónica importante escrita en 1615 por un indio hispanizado es la Nueva corónica y buen gobierno de Guamán Poma de Ayala. El texto no tiene un capítulo dedicado a las prácticas médicas tradicionales, pero sí tiene párrafos relativos a los curanderos, quienes son presentados como personajes con un importante rol social. Por ejemplo, en la página 826 [840] del manuscrito, Guamán Poma diferencia a los curanderos no idólatras o a las parteras de los otros sanadores denominados hechiceros, que sí podían oponerse a la "correcta práctica cristiana", con el claro objetivo de que puedan seguir ejerciendo su trabajo:

Los dichos yndios serojanos, barberos que curan y sangran y saben y conosen de las enfermedades y llagas, de las yeruas con que se an de curar y medicinas y purgas destos rreynos curan tanbién como un dotor o lesenciado de medecinas. $Y$ dizen que todas las enfermedades prosede de dos cosas que tiene los honbres, calor o frío en qualquier enfermedad, y las dichas mugeres beatas y comadres médicas que curan y ayudan a bien parir a las mugeres preñadas y algunas curan los desconsertados, las cuyunturas y otras enfermedades. A estos dichos yndios le ponen en pleyto el padre, corregidor o los propios yndios les llaman hechiseros, no lo ciendo cino cristiano. Antes conbiene para el $\underline{\text { seruicio de Dios y de su Magestad y bien de los pobres }}$ 
vndios y para que cure azogado; se le deue dar su mandamiento para curar y sangrar y que use oficio de barbero seruxano. El tauaco es ponsoña para calenturas y para frío, santa cosa poquito; ci es mucho cuese las entrañas. Mejor es en polbo por las narises. Esto de las medicinas no lo escribo porque no puedo más que esotro dotor y trauajo, salud de los honbres 200 años.

Garcilaso y Poma de Ayala no profundizaron en el estudio de los usos médicos de las plantas o productos naturales. Quienes sí lo hicieron fueron algunos jesuitas que publicaron textos sobre el reino natural de estas tierras y su uso curativo. La Compañía tuvo desde su origen un especial interés en evangelizar mediante el cultivo del conocimiento, tanto a nivel receptivo como expositivo. Por ello, sus integrantes dejaron testimonio amplio de las riquezas naturales del Perú y de la forma en que estas eran usadas para la salud. Así, al poco tiempo del arribo de esta orden al Perú (1568), uno de sus miembros, Joseph de Acosta (1540-1600), de tan solo 32 años, comenzó a registrar las características naturales y culturales del Perú andino. Fruto de ese trabajo iniciado en 1572, se publicó en 1590 la Historia natural y moral de las Indias, en la que Acosta da cuenta de las plantas americanas y sus propiedades médicas, en particular en el libro cuarto (la obra se divide en siete libros, los cuatro primeros destinados a la naturaleza indiana). La intención principal del autor, ciertamente, no fue la de dar cuenta de las prácticas médicas de los indígenas, pero como la cultura andina precolombina utilizaba como práctica curativa la dieta, es decir, el uso y consumo de productos alimenticios, son constantes las referencias a prácticas curativas. Por ejemplo, en el capítulo titulado "Del pan de Indias y del maíz" explica los usos medicinales de la chicha preparada con maíz tostado, que en los tiempos de Acosta (fines del XVI) era consumida tanto por indígenas como por españoles: "El modo más limpio y más sano y que menos encalabria es de maíz tostado; esto usan los Indios más pulidos y algunos españoles por medicina; porque en 
efecto, hallan que para riñones y urina es muy saludable bebida, por donde apenas se halla indios en semejante mal, por el uso de beber su chicha" (Acosta 2006: 192).

Siguiendo la ruta dejada por Acosta, destacó el padre jesuita Bernabé Cobo (1580-1657). Este arribó al Perú entre 1599 y 1600 , cuando Acosta ya no se encontraba en estas tierras ${ }^{2}$ y vivió aquí hasta 1630 en que viajó a México. Luego regresó en 1642 y se quedó en el país hasta su muerte en Lima en 1657. Cobo se ordenó en el Perú como sacerdote de la Compañía de Jesús en 1613, fecha alrededor de la cual debió iniciar sus estudios naturales (Mateos 1964: t. 1: XX), que reuniría luego en una obra mucho más monumental que la de Acosta: Historia del Nuevo Mundo. Es particularmente valiosa la primera parte de esta obra, que terminó de componerse en 1653 (fecha del prólogo) luego de 40 años de investigación. Según las propias palabras del autor, trata acerca de "la naturaleza y cualidades deste Nuevo Mundo, con las cosas que de suyo cría y produce" (Cobo 1964: t. 1: 5). Esta primera parte (de tres originales) es la única que se conserva intacta; se han perdido partes importantes de su obra estrictamente histórica relativas al Virreinato del Perú y México. Por esto es que Cobo tiene más fama como naturalista, aunque en sus descripciones naturales no deja de comentar prácticas humanas como ocurre en el Libro Cuarto de su obra, en donde consigna las aplicaciones curativas de productos peruanos, como es el caso de la hoy famosa maca. Cobo (1964: t. 1, p. 170) resalta las cualidades fertilizadoras que los indígenas le otorgaban, como hoy la sabiduría popular lo reconoce: "Su temperamento es muy caliente, y es común opinión que, con ser la provincia de Chincha-cocha, donde se da esta raíz, muy estéril y de tan frío y áspero temple, por mantenerse sus naturales con esta raíz no sólo no van a menos, como en las

2 Joseph de Acosta se fue del Perú en 1586 y llegó a España un año después luego de una estadía en México. 
demás provincias del Perú, sino que se multiplican de cada día más, para lo cual dicen tener virtud esta raíz. (El subrayado es del autor de este artículo).

Otras fuentes coloniales importantes son los documentos de extirpación de idolatrías, que contienen material sobre las prácticas medicinales tradicionales, ya que los curanderos eran personajes importantes y respetados en la sociedad andina y que -más allá de la diferenciación realizada por Guamán Poma- realizaban prácticas adivinatorias y curativas que por su componente mágico fueron tildadas de hechicería. Destaca el texto de Cristóbal de Molina Relación de fábulas y ritos de los incas, de 1573; el Tratado y relación de los errores, falsos dioses y otras supersticiones y ritos diabólicos en que vivian antiguamente los indios de Huarochirí de Francisco de Ávila, de aproximadamente 1598, que fue publicado por primera vez en 1966 con el título Dioses y hombres de Huarochirí; y la Extirpación de la idolatría del Pirú, de Pablo Joseph de Arriaga de 1621. Con base en este último documento, Nicholas Grifiths (1998: 142) afirma en su reconocido libro La cruz $y$ la serpiente que los extirpadores intentaron salvaguardar la práctica médica andina y "limpiarla" de sus "supersticiones", lo que se volvió una mecánica apoyada por la jerarquía eclesiástica:

Según Arriaga, el pecado de los curanderos nativos residía, no en las curas que aspiraban a hacer, sino en los métodos supersticiosos o idolátricos que empleaban. De hecho, tal era su respeto por su conocimiento de las hierbas nativas que proponía que los sacerdotes debían de proporcionar educación a los practicantes (los curanderos) y así eliminar cualquier sospecha de superstición. De esta manera podría sacarse provecho absoluto de sus habilidades sin el peligro de pecado. Esta postura fue adoptada por el arzobispo Villagómez. En sus instrucciones a su visitador general, escribía que, mientras los hechiceros y los ministros de la idolatría de indios no les debía de dejar de manera alguna 
curar a los enfermos, las actividades curativas de otros indios, cuya práctica se fundara en un conocimiento experto de las virtudes de las plantas, debían de ser aprobadas. (El subrayado es del autor de este artículo).

Entre los documentos administrativos coloniales, otra veta para explorar son los informes o visitas particulares, como es el caso de los textos del licenciado Polo de Ondegardo, entre los que destaca su informe Los errores y supersticiones de los indios, aparentemente redactado en 1559 cuando fungía de corregidor del Cusco, pero publicado en 1585 por interés del III Concilio Limense. Este texto se centra en analizar las creencias incas, incluidas las relacionadas con la salud. El capítulo X trata sobre "las hechicerías" y los curanderos que se dedican al "daño", "diestros en hazer confecciones de yeruas, y rayzes para matar al que las dán”. El capítulo XIII está dedicado a los "cvras y médicos" que aprendieron su oficio en sueños. Este último capítulo es poco profundo y explica más el oficio de las parteras o de las "hueseras" (Ondegardo [1585]).

Luego del gran interés por conocer y dar cuenta de las prácticas médicas andinas en el siglo XVI y en los comienzos del XVII, hubo un decaimiento en la investigación de este campo. Al parecer, luego de entender la actividad y el valor de los curanderos en la sociedad andina, con miras a la evangelización y al conocimiento histórico del mundo prehispánico, se dejó de tratar el tema casi por completo. Por ejemplo, el cronista satírico de la medicina peruana de la segunda mitad del siglo XVII, Juan del Valle y Caviedes (1990), no trata el tema de los curanderos o médicos tradicionales indígenas. Esto no quiere decir que la medicina tradicional andina perdiera importancia en el mundo colonial, ya que existen algunos testimonios aislados que dan cuenta de la importancia y vigencia del saber médico tradicional indígena. Así, tenemos conocimiento de la fuerte oposición en el claustro sanmarquino a la creación de cátedras de medicina en 1637, por 
considerarse que "los indios curan mejor que los médicos sanando a los que estos han desahuciado y que... curan muy acertadamente sin ser médicos"3; o de la importante difusión entre la élite limeña del uso de la corteza del árbol de la quina para curar la malaria, tal como el conocimiento ancestral andino lo sugería ${ }^{4}$. Por otro lado la "farmacopea" andina ya tenía gran influencia en las boticas de Lima. Así lo sugiere el inventario de una botica ${ }^{5}$ limeña de 1655 , en el que se observan productos andinos como la mencionada quina o el chamico. Esta presencia de productos de tradición indígena sigue vigente a lo largo del siglo XVIII, como lo demuestra el análisis del inventario de la botica del Colegio de San Pablo de la Compañía de Jesús. Según Uriel García Cáceres (2005: 27), este documento, consignado por Valdizán en su tercer tomo de La medicina popular peruana, constituye una "demostración del intercambio o mestizaje de la terapéutica médica" peruana.

A pesar de que la bibliografía sobre la medicina tradicional andina mengua a partir de la segunda mitad del XVII, existe material valioso en las causas judiciales. Así lo sugiere la reciente publicación de un documento de 1710 del Archivo General de Indias de Sevilla sobre el proceso judicial por hechicería contra el "indio" peruano Juan Vazquez. Este documento ha sido publicado íntegro en el libro de Luis Millones, Las

3 Opinión del Dr. Monzo de Huerta, catedrático jubilado de lengua quechua (Ugarte 1965: 9).

4 Esta práctica curativa andina ganó gran difusión a partir de la curación de las fiebres intermitentes de doña Francisca Henríquez de Ribera, esposa del virrey conde de Chinchón (1629-39). Luego de su curación, la condesa difundió el remedio entre la población local y, debido a esto, los padres jesuitas, los grandes boticarios de la época, comenzaron a enviar grandes embarques de la corteza a Europa.

5 Manuscrito colonial de propiedad de Uriel García. Véase GARCÍA, Juan del Valle Caviedes: Cronista de la medicina, p. 44 y nota 72. 
confesiones de don Juan Vaqquez. (IFEA, Fondo Editorial PUCP 2002), gracias a la labor paleográfica de Laura Gutiérrez. La información que presenta el documento es muy rica y se observa la gran aceptación del arte curanderil andino en Lima a inicios del XVIII, ya que la causa judicial tiene 24 testigos de la capital, que en su gran mayoría se refieren de forma favorable al curandero andino y cuentan cómo fueron tratados y curados por el sanador nacido en Cajamarca. Generalmente, el diagnóstico se hacía mediante el esputo del paciente y el pulso $^{6}$, mientras que en la curación se usaban hierbas andinas.

Otro ámbito de exploración interesante es el de los viajeros. Por lo revisado, lo que consignan respecto de la medicina andina es mínimo, pero es necesario hacer una investigación profunda. Por ejemplo, tanto La relación del viaje por el mar del sur de Amedée Frezier, de 1716, o el famoso texto de Jorge Juan y Antonio de Ulloa Noticias secretas de América, de 1747, pero publicado en 1826, solo contiene noticias del uso de la coca por los hombres andinos y nada más sobre la medicina propia del Perú.

Conforme avanza el siglo XVIII, el liberalismo ilustrado contribuye a que parte de la intelectualidad occidental peruana tenga un mayor interés por comprender la historia, naturaleza y cultura propias (influencia del nacionalismo incipiente). Esto queda claro al analizar las páginas de la principal publicación intelectual de la época, el Mercurio Peruano, revista académica publicada entre 1791-1795.

6 "Preguntado que quando toma el pulsso a los que padecen el achaque que le consultan como los conose sin averle dicho el enfermo el accidente que padece $i$ aviendole oido unas dos y tres vezes la explicacion de esta respuesta para su inteligencia dijo que cuando toma el pulsso al enfermo $i$ hecha la saliva en la palma de la mano conose como si lo estuviera viendo el accidente..." (Millones 2002: 104). En su estudio, Millones (p. 45) refiere que el curandero, mediante el pulso, podía detectar ladrones y hasta la ubicación de cosas robadas por ellos. 
El patriotismo de los mercuristas se manifiesta en los escritos de Hipólito Unanue sobre el mundo natural del Perú. Destaca su Botánica. Introducción a la descripción científica de las 'plantas del Perú', de 17917', una síntesis de los trabajos sobre especies vegetales producidos durante la colonia, con una propuesta más organizada influenciada por Linneo, en particular por su doctrina sobre la reproducción sexual en las plantas $^{8}$. Pero no se encuentra en esta obra mayores referencias a las prácticas curativas andinas. Otro trabajo importante es su Disertación sobre el aspecto, cultivo, comercio y virtudes de la famosa planta del Perú nombrada Coca, en la que toma en cuenta la sabiduría ancestral andina y hace una exposición de sus virtudes digestivas, sus beneficios para la circulación y la resistencia física, como disipador de cuadros melancólicos y como supresor del hambre. Estas virtudes llevan a Unanue a sugerir que esta planta peruana debía reemplazar al té o al café en el mercado mundial, posición bastante peruanista. A pesar de esto, se nota en los textos de Unanue así como en el texto Necesidad de la historia natural cientifica, de Francisco González Laguna ${ }^{9}$, un menor interés por entender la lógica andina acerca del uso médico de las plantas. Influidos por el pensamiento occidental moderno, muestran una mayor indiferencia hacia el saber andino que sus antecesores naturalistas del siglo XVI o XVII.

7 Mercurio Peruano, t. II, n 43-44, 29 de mayo y 2 de junio de 1791.

8 Naturalista sueco (1707-1778), autor de una clasificación de plantas, es considerado el creador del método científico en el estudio de la historia natural.

9 Mercurio Peruano, t. X, n 316, 317, 318 y 319 del 12, 16, 19 y 23 de enero de 1794. 


\section{La República: Del desinterés a la revaloración del saber curativo andino}

Con el advenimiento de la República, la indiferencia hacia la práctica médica tradicional andina, que ya se encontraba presente en la centuria iluminista (siglo XVIII), se acentuó y por ello no encontramos mayores referencias al respecto hasta mediados del siglo XIX, cuando un extranjero nos hizo recordar el valor de la curación con plantas andinas. Nos referimos al italiano Antonio Raimondi (1826-1890), quien no solo valoró el saber ancestral curativo mediante sus observaciones, sino que tuvo la suerte de apoyarse en su propia experiencia. Según su biógrafo Stefano Varese, su primera experiencia con la curación indígena se registró en Tingo María en 1857 cuando "sana de un reumatismo a la rodilla con el uso de una hierba", lo que da origen a su interés por las plantas medicinales basadas en el conocimiento tradicional peruano (Varese 1965: 27). La voluminosa obra de Raimondi tiene referencias a prácticas médicas indígenas, obviamente, debe destacarse su obra máxima El Perú, publicada en seis tomos entre 1874 y 1913 . Un valor particular de la obra de Raimondi es que es quizás el primero que intenta darle un valor científico moderno a la sabiduría curativa andina. Es lo que ocurre, por ejemplo, cuando se encuentra en el norte del país con una práctica médica extraña: el uso de las heces de aves Steatornis o guácharos para curar las fiebres tercianas. Luego de analizar a estas aves, el sabio italiano llegó a la conclusión de que la razón científica de ese uso medicinal era la alimentación de las aves con las semillas de una planta del género Nectandra que contiene un alcaloide usado en Inglaterra junto con nuestra quinina para curar esas fiebres. Como dice Varese: "Otra vez la medicina de los indios se demuestra válida y racional, no supersticiosa como había creído Humboldt" (Varese 1965: 44). Por este tipo de experiencias Raimondi revaloriza el saber curativo 
andino en una época en la que el positivismo comteano y el darwinismo social influían en los dirigentes peruanos ${ }^{10}$. La admiración de Raimondi hacia el indio y su cultura médica quizás no se haya igualado hasta ahora, aunque en la actualidad existe más apertura hacia el saber andino. La admiración al indígena en Raimondi queda clara en una célebre frase de El Perú de 1874: "[...] aún los dichos, tradiciones y hasta preocupaciones de la raza indígena, que a primera vista parecen absurdos, sometidos al crisol del criterio se descubre casi siempre en ellos alguna verdad, que solamente ha sido mal interpretada".

Raimondi falleció en 1890, pocos años después de concluida la guerra con Chile (1879-1883). Esta infausta derrota motivó en los peruanos una mirada hacia adentro, que obligó a las élites políticas y culturales a repensar el valor de lo indígena y la importancia de una mayor comprensión de su cultura y mentalidad para construir realmente una nación unida y fuerte. Paralelamente, la ciencia médica avanzaba y los estudios médicos se modernizaban y formalizaban. Gracias a estas dos fuerzas, un interés renovado por lo indígena (desde inicios del siglo XX) y la modernización de la enseñanza médica, aparece en la escena historiográfica nacional la figura del médico Hermilio Valdizán (1885-1929), quizás el más grande investigador de la medicina peruana de toda la historia. Aún falta una biografía sobre este insigne médico nacido en Huánuco, que nos permita valorar su obra en su real medida. Valdizán tuvo temprano interés por historiar la medicina nacional. Así, en 1911, con 26 años y a solo dos años de graduarse como bachiller en medicina, publicó sus

10 Esta es la época en la que Manuel Pardo, el líder civil del Perú de la segunda mitad del siglo XIX, fomentaba, tanto como ciudadano común así como presidente de la república, la inmigración extranjera como requisito casi primordial para lograr el progreso del país. 
dos primeros libros: La Facultad de Medicina de Lima 1811 1911 y Nuestra medicina popular. Apuntes para su historia. El primer texto es un homenaje por los 100 años de la Escuela de San Fernando que inició la formación en medicina durante la colonia. Si bien el interés del autor es el desarrollo de la medicina occidental, el texto menciona la importante labor médica realizada por los indígenas en la colonia, incluso a los españoles y criollos. Por ejemplo, hace referencia a la crónica del Inca Garcilaso y a la curación de heridas de guerra con estiércol de auquénido a las tropas rebeldes de Gonzalo Pizarro y reconoce la presencia de curanderos indígenas no solo en los Andes o en el ámbito rural, sino también en las urbes: "Junto a estos profesores de medicina y cirugía, autorizados por los cabildos (...) vivían en la Capital del Virreynato muchos curanderos: algunos naturales, que, seguramente ejercían los conocimientos trasmitidos por sus antecesores [...]" (Valdizán 1911a: 21c).

En el segundo texto mencionado, el autor relata diversas anécdotas, aunque no queda claro si todas son de su vida, en relación con la medicina tradicional peruana, y busca, como lo dice en la presentación, que los doctores occidentales al acercarse a la serranía "conozcan algunos secretos de la medicina popular" y puedan desempeñarse mejor en la región: "Es la voz de alerta del camarada que comprende los peligros que significa para el médico su total ignorancia de los prejuicios del público cuya salud le ha sido confiada" (Valdizán 1911b: 1).

En el primer capítulo, titulado "El susto y el 'shogpi"”, cuenta la historia de una madre que llevó a su hijo de un año, enfermo de susto, para que el doctor occidental recién llegado al pueblo lo curara (Valdizán 1911b: 2). Narra el descontento de la madre al observar que el admirado médico occidental no sabía curar el susto y de su decisión de llevar al hijo donde una curandera para que le aplique el shogpi (curación 
del susto). Al final de esta anécdota, comenta el fracaso del tratamiento tradicional de lo que para Valdizán era desnutrición. El capítulo 3 titulado "La zooterapia" cuenta anécdotas sobre el uso de productos animales (incluidos los orines y heces de hombres) para curar determinadas enfermedades (1911b: 16-21) y en el capítulo 4 titulado "El chamico y su obra" comenta sobre una poción usada en la serranía para "atrapar" a un ser querido (1911b: 22-25). A lo largo de estos relatos queda clara la mirada sorprendida y negativa de Valdizán respecto a estas prácticas y creencias. Por ejemplo, en el caso del susto señala: "Nuestro pobre enfermito resistió el baño de tierra y aún pareció experimentar una ligera mejoría. Pero fue una mejoría engañosa. La pequeña lámpara se extinguió. En presencia del cadáver del niño la curandera excusa plenamente su fracaso. La han llamado tarde. Y la madre cree. ¡Feliz ella! (...) No he vuelto a sonreírme ante un diagnóstico de susto. Y ha sido así, que he tratado en muchos casos los estados patológicos que derivan de una insuficiente nutrición” (1911b: 5).

No sabemos cómo luego Hermilio Valdizán pasó a tener una visión más neutral o menos crítica de las prácticas médicas andinas. El giro queda claro ya en 1922, cuando junto a Ángel Maldonado publica La medicina popular peruana, que aún hoy es considerada como la principal obra sobre este tema. Esta publicación en tres tomos es probablemente la más importante de la producción de Valdizán. El primer tomo está dedicado a una descripción de las creencias y prácticas médicas del Perú en distintas épocas recopiladas de crónicas coloniales y relatos orales del siglo XX. No existe un orden importante, pero sí mucho material que debería ser clasificado y procesado en el futuro. El tomo II es una larga relación de la farmacopea naturista andina del siglo XX (por lo que se incluyen plantas traídas por los españoles), una recopilación que según Uriel García (2005: 26) aún ahora es difícil de superar. Por ejemplo, respecto a la granadilla, una fruta tan 
apreciada hoy, se cita al cronista colonial Calancha para explicar que la flor en vino era usada por los indios para sanar el "mal de sangre"; luego se consignan sus aplicaciones contemporáneas (inicios del s. XX) según las diversas regiones del país: "Los frutos se emplean en la alimentación y como preventivo de la fiebre amarilla (norte); el cocimiento de la cáscara del fruto, en la tos rebelde (Ica); el cocimiento de las hojas con verbena en el paludismo (S.P.); en cocimiento en la espermatorrea y como estomacal y también para baños de asiento (Loreto); la tintura como febrífuga (Lima); la infusión o tintura de las hojas como febrífuga (Ica)" (Valdizán y Maldonado 1922: 259).

El tercer tomo constituye un aporte documental valioso para los investigadores del presente y futuro, ya que es una colección de cuatro documentos de fines del siglo XVIII e inicios del XIX sobre la principal botica de fines de la colonia en 1770 (botica del Colegio San Pablo de Lima); dos recetarios populares del XVIII (uno de ellos inspirado en la obra de Cobo) y la relación de un viaje al interior del país de 1821 (Larecaja) con una descripción de los productos naturales encontrados en el trayecto y su uso curativo.

La principal crítica que se debe realizar a esta obra monumental sobre el saber curativo tradicional es el poco análisis y sistematización realizado por los autores. A pesar de ello, es considerada por Uriel García (2005: 25), uno de los principales historiadores de la medicina peruana, como "la piedra angular de los estudios etnológicos, antropológicos e históricos de la medicina popular peruana”.

Con La medicina popularperuana no acaban los aportes de Valdizán a la historia de la medicina tradicional. Dos años después de publicar esa gran recopilación, Valdizán terminó de escribir su Historia de la medicina peruana (1924), cuyo título es engañoso porque solo llegó a tratar la historia de la medicina 
precolombina. Este libro, por razones que desconocemos, solo llegó a publicarse en 1944 y es el que contiene mayor interpretación y reflexión de todos los textos de Valdizán. A pesar de ello, tampoco logra un análisis claro sobre la evolución de la medicina prehispánica, sino que realiza una síntesis descriptiva de las prácticas médicas de los incas, basado en las crónicas coloniales. La obra está dividida en temas como obstetricia, pediatría, cirugía, oftalmología, entre otros (Valdizán 2005 [1939]).

Es importante hacer notar que la obra más importante de Valdizán fue realizada durante el oncenio de Leguía (19191930), una etapa en la que se revalorizó al indio. No sabemos en qué medida el indigenismo pudo haber influido en este autor, pero sí tenemos certeza de que para su obra máxima, La medicina popular peruana, contó con el apoyo estatal en la persona del ministro de Fomento, que financió la obra. Así lo dejan saber tanto Valdizán como Maldonado (1922: t. 1, III-IV) en su prólogo:

Primera palabra obligada de este libro es la expresión de gratitud de sus autores al apoyo decidido que para darlo a la publicidad han hallado en el señor Ministro de Fomento, Doctor Lauro Ángel Curletti. Ha sido merced a la cultura del señor Ministro de Fomento; merced a sus entusiasmos de apoyo a la obra nacionalista que, dentro del marco de la modestia, representa este libro, que él puede ver la luz pública, después de algunos años de espera impuesta por las dificultades que hoy en día rodean al problema editorial y por aquellas otras dependientes de la parquedad de recursos económicos de los autores.

Valdizán dejó escuela entre los médicos peruanos, razón por la que luego de su fallecimiento en 1929, las publicaciones sobre historia de la medicina (de las que él fue prácticamente el pionero) continuaron. Valdizán dejó discípulos porque tuvo la clara intención de difundir su pasión por la historia 
médica peruana, tal como lo hacía en sus tertulias dominicales, de las que el Dr. Juan Francisco Valega (1935) ha dejado testimonio. Quienes continuaron con su labor histórica fueron en particular los doctores Juan B. Lastres y Carlos Enrique Paz Soldán, aunque para el caso de la medicina tradicional peruana debemos destacar, en especial, el trabajo del primero. Lastres, debido posiblemente a su labor como primer docente de la cátedra de historia de la medicina que comenzó a dictar en 1946, buscó organizar mejor los cocimientos acerca de la medicina prehispánica y colonial, que son las épocas de génesis de nuestra actual medicina tradicional. ${ }^{11}$ Esta labor la reunió en los tres tomos de la Historia de la medicina peruana, de 1951, quizás la obra más importante en el campo de la historia médica peruana hasta hoy. Para la historia de la medicina tradicional andina en el Perú son particularmente importantes los dos primeros tomos dedicados a la medicina incaica (vol. 1) y a la medicina de la colonia (vol. 2). En esta obra hay un análisis más profundo que en los textos de Valdizán, pero el interés en la medicina tradicional disminuye, ya que la obra tiene el objetivo de describir el desarrollo de la medicina científica en el país. A pesar de ello, hay citas y referencias muy valiosas.

Luego de la obra de Lastres, muchos médicos han seguido el enfoque descriptivo de la historia de la medicina apoyados en las obras de este y en las de Valdizán. Pero el interés por la medicina tradicional se redujo, en parte por la mayor difusión de la medicina moderna en el Perú -debido a la mayor penetración de ella en el interior del país y al arribo de perua-

11 La cátedra de historia de la medicina, a pesar de que obliga a Lastres a organizar de forma más sistemática su conocimiento sobre la medicina prehispánica y colonial, no es la causa de su interés en la medicina de aquel tiempo. Años antes ya había publicado un texto sobre la medicina de inicios del XVII, titulado La medicina en la obra de Guamán Poma de Ayala (Lima: Imprenta del Museo Nacional, 1941). 
nos de provincias hacia los centros modernos como Lima-, así como por el decaimiento, en el propio gremio médico, del interés por analizar su propia historia al percibir que su profesión se encontraba ya consolidada. Esta es la opinión de Marcos Cueto (1989: 25), uno de los principales historiadores de la medicina de los tiempos actuales:

Luego de 1960 existieron muchos autores que escribieron muy poco (...) Asimismo, las pocas personas que hicieron historia de la medicina y de la ciencia mantuvieron las características principales del modelo histórico inaugurado por Valdizán. (...) El original esfuerzo de Valdizán por mostrar las realizaciones médicas nativas fue congelado en una genealogía de nombres, hechos e instituciones. Otra razón para el declive de la historia de la medicina en el Perú es el hecho de que la construcción de una historiografía oficial dejó de ser relevante para la profesión médica cuando ésta se encontraba mejor establecida. Después de los años cincuenta, la medicina formal empezó a desplazar, por lo menos en las áreas urbanas, a los practicantes informales de la medicina. Entonces, ya no fue tan importante contar con el soporte cultural que brindaba el trabajo de Valdizán. Los médicos empezaron a reafirmar su identidad profesional a partir de su especialidad. Asimismo, al enfatizar la imitación de los patrones de trabajo de los países desarrollados, se dejó de lado las tradiciones locales.

Esta declinación por la historia de la medicina la encuentra Cueto presente hasta los días en que se publica su libro, del 98 que se acaba de extraer unos párrafos (1989). En realidad, en la década de 1980 algunos médicos reiniciaron la investigación con gran fuerza. Entre ellos destaca el Dr. Fernando Cabieses (1920-2009), neurólogo interesado en la antropología médica, que revaloró los trabajos de Valdizán acerca de la medicina tradicional y continuó su obra. Cabieses ya había mostrado en 1960 su interés por la medicina peruana tradicional al publicar un estudio realizado junto a Juan B. Lastres sobre las trepanaciones craneanas prehispánicas y luego en 
1974 publicó Dioses y enfermedades: la medicina en el antiguo Perú, un texto que recopiló en dos volúmenes lo que se conocía sobre la medicina prehispánica o tradicional. Es a partir de la organización de los dos primeros congresos mundiales de medicina tradicional (1979 y 1988), organizados en nuestro país con la presidencia de Cabieses, que se fortalece el interés por la medicina popular o tradicional en el ámbito médico, al menos así lo considera la principal publicación de la historia de la medicina nacional de los últimos años (Salaverry (editor) y Delgado Matallana (compilador) 2000), mediante el artículo de Manuel Fernández Ibarguen:

(El Primer Congreso de Medicina Tradicional) Se llevó acabo en Lima en 1979 bajo la inspiración del Prof. Carlos Alberto Seguín y lo presidió el Dr. Fernando Cabieses, esta cita ha sido el punto de partida para que los profesionales de las ciencias de la salud humana fueran tomando algún interés por la Medicina Tradicional; asistieron a este Congreso delegados de 23 países, resultando un éxito, hasta entonces la Medicina Tradicional estaba separada, casi ignorada por la profesión médica, luego en los años sucesivos fue incrementándose el interés por los aspectos positivos de la Medicina Tradicional (v. 2, p. 1110).

Según Fernández Ibarguen (v. 2, p. 1108), este mayor interés por nuestra medicina tradicional se debe a la nueva posición de la Organización Mundial de la Salud respecto a las medicinas tradicionales del mundo, a partir de la Conferencia Internacional de Alma Ata del año 1978. En esa conferencia la OMS consideró a la medicina tradicional como una aliada, especialmente en los países del tercer mundo, donde los servicios de la salud eran deficitarios, lo que motivó un mayor interés de parte del Estado peruano por la investigación en este campo. Debido a esto, Fernando Cabieses en alianza con el Dr. Manuel Fernández Ibarguen comenzaron las gestiones para fundar el Instituto de Medicina Tradicional, lo que se logró en abril de 1990, a fines del gobierno de Alan 
García Pérez, quien mediante el Decreto Legislativo $n^{\circ} 584$ dio la Ley de Organización y Funciones del Ministerio de Salud, que incluía la investigación y fomento de la medicina tradicional. El artículo 31 señala al respecto: "Artículo 31.- El Instituto Nacional de Medicina Tradicional es el organismo encargado de coordinar y proponer la política de medicina tradicional, así como desarrollar la investigación, docencia, normación (sic) y la integración de la medicina tradicional con la medicina académica para contribuir a elevar el nivel de salud de la población".

Desde su fundación hasta el año 2001, el INMETRA (Instituto Nacional de Medicina Tradicional) fue dirigido por Cabieses, tiempo en el que se comenzó a publicar la serie de Cuadernos de Medicina Tradicional que llegaron a ser, hasta donde conocemos, 24 números. Estos cuadernos fueron elaborados por el antropólogo Hugo E. Delgado Súmar y reunidos al parecer en una publicación del año 2000 del INMETRA. Se ha accedido a los cuadernos de forma individual (a los primeros 14 números) en la Biblioteca Central de la Pontificia Universidad Católica del Perú. Estos cuadernos no tienen fecha, aunque sí una numeración correlativa que indica que forman parte de una obra mayor. Constituyen una fuente sistematizada de la medicina tradicional andina, que enfatiza la comprensión de la lógica de los médicos tradicionales y no tanto, como antes, sus aplicaciones o prácticas concretas. Por ejemplo, en el primer número Salud y enfermedad en el mundo andino se explica que existen tres aspectos de la enfermedad para los hombres de los Andes: individual, grupal y ambiental. En el plano individual, las causas de la enfermedad son tres: a) excesos, en especial en el régimen alimenticio; b) trasgresión de las normas de reciprocidad con los dioses y c) accidentes fortuitos (Delgado Súmar s.f.: 1112). La preocupación por la lógica o cosmovisión médica de los hombres andinos es una característica reciente que debe ser destacada. Ello también está presente en la obra 
de Cabieses de los últimos años y tiene que ver con el auge de la antropología médica en el mundo, que ha permitido colocar a la comprensión de la cultura de cada pueblo cono un elemento clave para ejercer la sanación. Al respecto, Fernando Cabieses (1985) al comentar en el prólogo de la edición facsimilar de La medicina popular peruana de Valdizán y Maldonado (1922), a quienes considera como pioneros de la antropología médica mundial, menciona:

El resurgimiento actual de los factores causales de enfermedad dentro del amplio campo de las ciencias sociales ha puesto de manifiesto un nuevo enfoque patogénico: la presencia de elementos culturales, comunitarios, políticos, psicosociales que provocan trastornos funcionales, psicológicos y fisiológicos que atentan contra la salud y el bienestar de una población. Todo lo que rodea la problemática de la salud (...) se encuentra entretejido con la manera en la que cada grupo humano percibe su propio mundo y pertenece al ámbito de los valores sociales que estudia la Antropología.

Por esta nueva visión, Cabieses buscó actualizar el trabajo de Valdizán y Maldonado de 1922, en particular, la larga relación del uso curativo de los productos naturales peruanos descrita en el tomo II, lo que dio origen a sus Apuntes de medicina tradicional, la racionalización de lo irracional (Lima: A y B Editores, 1993). Por otro lado, Cabieses tiene una larga trayectoria como investigador del uso tradicional de la hoja de coca y defensor de esta práctica que comenzó con su tesis de 1946, en la que demostró que el uso tradicional indígena de la coca no producía narcodependencia ni daño alguno.

El mayor interés de los médicos en la medicina popular andina está ligado también a ciertos giros de tipo posmoderno en la ciencia médica, como la influencia de la mente y las emociones en el cuerpo físico, lo que en la cultura médica andina tradicional peruana siempre había sido considerado (el caso 
más típico quizás sea el del susto). Por ello, se han difundido prácticas a nivel mundial como la de los "doctores bola roja" que buscan relajar y hacer reír a los pacientes para causar una mejoría en su salud. Por otro lado, el fortalecimiento político-económico de China ha llevado a la difusión de su cultura médica tradicional, como es el caso de la acupuntura, lo que a su vez ha permitido una mayor tolerancia de parte de los médicos occidentales modernos hacia las prácticas tradicionales en general. Inclusive la OMS publicó en el 2002 su primera estrategia global sobre medicina tradicional, debido no solo a la sobrevivencia de prácticas tradicionales en muchos países (particularmente los menos desarrollados), sino también al mayor interés por la denominada medicina complementaria o alternativa (MCA) en los países desarrollados:

En muchos países desarrollados, el popular uso de la MCA está propulsado por la preocupación sobre los efectos adversos de los fármacos químicos hechos por el hombre, cuestionando los enfoques y las suposiciones de la medicina alopática y por el mayor acceso del público a información sanitaria. Al mismo tiempo, la esperanza de vida más larga ha dado como resultado un aumento de los riesgos de desarrollar enfermedades crónicas y debilitantes tales como las enfermedades coronarias, el cáncer, la diabetes y los trastornos mentales. Para muchos pacientes, la MCA parece ofrecer medios más livianos de tratar dichas enfermedades que la medicina alopática (OMS 2005: 2).

En nuestro país, en los últimos años se ha reforzado la incorporación de la medicina tradicional a los sistemas de salud pública, al menos con hierbas de uso tradicional ${ }^{12}$. Esto ha

12 Información personal de familiares y amigos que hacen uso de los sistemas públicos de salud. Además, conocemos la práctica de acupuntura en el Hospital María Auxiliadora, donde en el 2006 se creó un Departamento de Medicina Alternativa y Complementaria. Véase al respecto: 
influido en los médicos dedicados a la investigación histórica y se refleja en una publicación de inicios del presente siglo, que logró reunir a los principales conocedores de la historia de la medicina peruana de la actualidad. Así, en la mencionada Historia de la medicina peruana del siglo XX (Salaverry (editor) y Delgado Matallana (compilador) 2000: 2 v.), que es una suerte de continuación de la Historia de la medicina de Lastres, que llegó hasta fines del XIX, se incluyen artículos de diversas tendencias curativas (no solo de la medicina occidental moderna académica). Por ejemplo, en el tomo II hay artículos sobre acupuntura y medicina tradicional. Este último reviste especial interés para nuestra exploración porque constituye un buen recuento de la práctica médica tradicional y de las investigaciones realizadas sobre ella hasta inicios del siglo XXI. El artículo proporciona una lista y reseña de la obra de los principales investigadores de la medicina tradicional de los últimos años, entre los que destacan antropólogos como el italiano Mario Polia o el ya mencionado Hugo E. Delgado Súmar (Fernández Ibarguen 2000: v. 2, p. 1117).

La producción de los antropólogos respecto a la medicina tradicional es amplia y variada, y se da principalmente a partir de 1950 hasta la actualidad. Es probable que el autor más prolífico sea Mario Polia, quien se ha dedicado a investigar a los curanderos de la costa norte, en especial a los de Piura desde la década de 1970. Ha escrito libros y artículos, entre los que destacan: Las lagunas de los encantos: medicina tradicional andina del Perú septentrional (1988); Cuando Dios lo permite: encantos y arte curanderil (1994); Despierta, remedio, cuenta...: adivinos y médicos del Ande (1996) y La sangre del cóndor: chamanes de los Andes (2001). En este último texto, Polia (2001: 23) toma una actitud diferente de la de sus anteriores libros y se presenta como un observador activo, como el investigador compro-

http://blogs.elcomercio.pe/ejecutivas/2010/06/zenaida-mateos-todaenfermedad.html 
metido que fue desde que comenzó sus investigaciones en Piura y participó como paciente y aprendiz de chamán en los diversos rituales curanderiles. Por ello, cuenta con extraordinaria claridad el proceso de toma del cactus del San Pedro o la curación del susto de una mujer debido a que su "sombra" (alma) se quedó en una corriente de río: "A través de las quebradas te estoy guiando. A través de las quebradas te estoy llamando, para que te levantes y vengas aquí (...) Con el poder de mis toros te voy jalando. Con el poder de las varas de la selva te llamo. Aquí debes venir. Con el poder de la cruz y de mis imanes te jalo a través de campos y abismos, por remolinos de viento y de agua" (p. 257).

Este llamado de regreso a la "sombra" (alma) es similar a otros rituales de tiempos coloniales o prehispánicos, que describen los documentos virreinales, y es sorprendente apreciar la continuidad de la práctica en el tiempo. En el artículo Supay de Gerald Taylor (2003) se explica que una de las primeras acepciones de "supay" es el de "sombra" o "alma" y también se describen prácticas curanderiles que piden a "supay" que regrese al cuerpo del hombre enfermo. Con lo dicho, queda claro que se necesita una mayor colaboración entre los conocimientos históricos y antropológicos para entender mejor el fenómeno de la medicina tradicional en el tiempo. En el caso de Polia, el apoyo en los documentos históricos es recurrente. Por ejemplo, al describir la venta de collares de ishpingo en los puestos que se abren en torno a la fiesta del señor Cautivo de Ayabaca, todo un acontecimien-

104 to en la región, hace un recuento amplio de las referencias coloniales respecto al poder curativo de este producto de la flora peruana. Polia dedica más de una página de notas a esta planta en las que cita a Polo, Guamán Poma, Arriaga, Murúa, González Holguín y Cobo. Polia (2001: 240) nos cuenta, por ejemplo, que el ishpingo mezclado con molle y salvia "hace fecundas las mujeres estériles". 
Otro importante antropólogo de las últimas décadas es el ya mencionado Hugo Delgado Súmar, quien tuvo una producción prolífica de textos a fines de la década de 1980. Delgado se dedicó fundamentalmente a estudiar a los curanderos de Ayacucho, siendo una de sus primeras importantes obras la Medicina tradicional en Ayacucho. Testimonio, en la que describe el pensamiento y práctica médica tradicional de Guillermo Huayllani Raymundo, un curandero huancavelicano que ejerce su oficio en Ayacucho. El texto se inicia con la historia personal del curandero y cómo empezó esta labor (1988: 27-28). En el capítulo 2 titulado "Yo... curandero" trata el aspecto mágico de la labor, la relación que debe existir con las divinidades andinas para poder curar y el diagnóstico mediante el cuy o la consulta de naipes. Luego, el texto se dedica a explicar las prácticas sanadoras. En el capítulo 3 explica las curaciones para cada tipo de dolencia, clasificadas por regiones corporales (hígado, corazón, estómago, etc.), mientras que en el capítulo 6 clasifica las curaciones de los males sutiles como el susto, ojeo, pacha, puquio, etc. El texto termina con una farmacopea tradicional y la reseña de productos vegetales, animales y minerales.

Delgado Súmar, al parecer, se introdujo en el mundo curandero ayacuchano con esta obra, ya que luego de ella publicó pequeñas obras editadas por la Universidad San Cristóbal de Huamanga, la mayor parte de ellas en los Cuadernos de Investigación de la Facultad de Ciencias Sociales. Destacan Rituales y procedimientos curativos en Ayacucho, La tuna en la medicina tradicional y Los curiosos en la medicina tradicional, publicados en 1989.

Luego de su experiencia en Ayacucho, Delgado Súmar a inicios de la década de 1990 comenzó a colaborar con el recientemente creado Instituto Nacional de Medicina Tradicional, a lo que ya hemos hecho referencia. Luego, fruto de sus estudios de maestría en la PUCP es su obra antropológica 
sobre la medicina tradicional, La medicina tradicional en Lima: Inmigrantes de segunda y tercera generación (1998), que constituye un análisis de las prácticas de la medicina tradicional en Lima en los albores del siglo XXI, luego del proceso de mestizaje cultural que se produjo por las migraciones.

En el ámbito de la disciplina histórica, en los últimos años hay interés por historiar la medicina tradicional peruana. Ello se debe a la influencia de la antropología y de los estudios interdisciplinarios en los historiadores de fines del siglo XX (debido a la pérdida de fuerza de las tendencias positivistas). Destaca el trabajo de Luis Millones Santa Gadea, historiador-antropólogo interesado en el universo mental y religioso de los hombres andinos que, como hemos visto en este texto, es un tópico ligado a la práctica de la medicina tradicional. A lo largo de su ruta historiográfica, Millones ha publicado textos relacionados con este tema centrado en la costa norte. El primer texto al respecto es Alucinógenos y shamanismo en el Perú contemporáneo, publicado con Mario Chiappe y Moisés Lemlij en 1985; luego En el nombre del Señor: shamanes, demonios y curanderos del norte del Perú, editado con Moisés Lemlij en 1994, y Entre Dios y el diablo: magia y poder en la costa norte del Perú, texto editado con Tatsuhiko Fujii y Hiroyasu Tomoeda en el 2004.

A estos textos se debe sumar uno que ya comentamos, Las confesiones de don Juan Varquez. (2002), que es un texto histórico por tratarse del análisis de una causa judicial contra un curandero indio a inicios del siglo XVIII. Sin embargo, Millones recurre a su bagaje cultural antropológico y le da mayor valor a su libro. Por ejemplo, al reflexionar sobre cómo es San Juan, el santo que se le aparece al curandero en la visión-sueño por la que recibe su don sanador señala:

Es interesante la elección de San Juan como el personaje de la visión divina que instruye al curandero. El apóstol de 
Cristo no suele aparecer en los juicios de idolatrías ni en las persecuciones de la Santa Inquisición de las colonias americanas. Otros son los referentes o menciones de la cristiandad que fueron asumidas por las sociedades indígenas, tampoco aparece en las informaciones etnográficas que se refieren al mundo de los curanderos, siendo San Cayetano y Santa Rosa los que reciben mayores invocaciones en las "mesas" o sesiones de curación del área andina. A estos santos se suman los santos regionales sean o no mencionados por la Iglesia católica (el Cristo Cautivo de Ayabaca, la Cruz de Chalpón, etc.). Lo más probable es que Vazquez se sintiera impresionado por tratarse del santo que le dio su nombre $[\ldots]$ (p. 45).

En esta última cita observamos lo valioso que significa tener un amplio conocimiento histórico y antropológico para interpretar la información sobre el curanderismo, sea del presente o del pasado. Lastimosamente, el número de historiadores, antropólogos o médicos con este bagaje de conocimiento es escaso en la actualidad (quizás Polia y Millones sean los de mayor preparación por los años que están explorando el tema). Debemos comprender que para abordar bien este tema se necesitan conocimientos acerca de la religiosidad, cosmovisión y medicina andinas; así como conocimientos de otras culturas tradicionales que amplíen la capacidad comparativa. Como sobre nuestra medicina aborigen los peruanos del pasado prehispánico no dejaron nada escrito, quizás debamos apoyarnos en medicinas tradicionales de otros lugares del mundo que tengan un registro escrito para así interpretar tanto los documentos coloniales y republicanos, como las prácticas actuales. Digo esto por una experiencia personal que deseo compartir al cerrar este breve trabajo.

A fines de los años ochenta e inicios de los noventa, un familiar cercano estuvo enfermo gravemente. Luego de seguir un tratamiento alopático y de no recuperar la salud, tomó la decisión de autocurarse aplicando los conocimientos natu- 
ristas que tenía (tanto por herencia familiar como por lecturas personales). Entre las cosas que aplicó fue importante la medicina tradicional de la India antigua o ayurveda. En pleno proceso de autocuración, le recomendaron a una experta curandera andina proveniente de la zona de Mala, a pocos kilómetros al sur de Lima. La curandera era una famosa partera sanadora de la región que ordenaba el aparato digestivo mediante masajes y oraciones. La señora, de nombre Espirita, fue recurrentemente a casa de este familiar y le ayudó a superar las dolencias que tenía en la región suprarrenal, pero al mismo tiempo le enseñó un mecanismo de diagnóstico mediante la toma del pulso, que según mi familiar era la explicación práctica al conocimiento teórico que él había revisado en los textos de la medicina ayurvédica india ${ }^{13}$. Esta experiencia personal me anima a sugerir que sería muy beneficioso para la investigación sobre la medicina tradicional peruana que se realicen más análisis comparativos con otras prácticas médicas tradicionales. Es probable que así surjan

13 Entrevista al licenciado Rubén Devoto Peralta (Lima, 17 de mayo del 2015), administrador de empresas, que desde los años noventa se dedica a la práctica de la medicina ayurvédica en el Perú. El Lic. Devoto ha publicado, en coautoría, Los tres principios vitales (Lima: Centro Cultural Védico, 1993); y Ayurveda. Principios básicos (Lima: Universidad Ricardo Palma, 2007). Según Devoto, la curandera Espirita le explicó que el primer pulso debía sentirse como una serpiente (energía vata en ayurveda); el segundo, como un sapito (pulso de la energía pitta para la India) y el tercero, como un patito nadando suavemente en el agua (pulso de la energía kapha en ayurveda). Estas descripciones son similares a las del ayurveda, en palabras del Lic. Devoto. Además, comenta que la práctica de la curandera y sus explicaciones le ayudaron a desarrollar la sensibilidad para tomar el pulso adecuadamente según la tradición ayurvédica. Este paralelismo se debería investigar más. Devoto afirma que la curandera le mencionó que en el pulso podía "mirar" accidentes del pasado, al estilo del curandero Juan Vasquez hace 300 años. Esto demuestra que hay prácticas, saberes y mentalidades ancestrales que están plenamente vigentes y que la antropología debe dar a conocer. 
preguntas nuevas y que pueda profundizarse en el conocimiento médico tradicional de nuestro país.

\section{Epílogo}

En los tiempos posmodernos existe una revalorización de los saberes tradicionales, debido a una multiplicidad de razones, como el cuidado ecológico, la sostenibilidad económica, la salud, etc. En el campo médico ocurre lo mismo; existe gran interés en las medicinas autóctonas por su poder preventivo, por ser menos invasivas y por contar con recetas milenarias que han mostrado eficacia.

Este artículo busca contribuir al conocimiento de la medicina tradicional peruana -campo poco trabajado por la historiografía peruanista- no solo por sus implicancias médicas, sino también como un medio para fortalecer nuestra identidad nacional, al permitir valorar más y mejor la cultura autóctona peruana.

Para cumplir este objetivo, he analizado de forma sucinta las principales fuentes que se conocen sobre la medicina andina. La documentación revisada ha permitido observar el gran respeto, y hasta admiración, que tuvieron los cronistas españoles por los médicos andinos. Ellos fueron los primeros en dejar testimonio escrito sobre la medicina tradicional en el siglo XVI. En ese mismo siglo, se produjeron también algunos textos académicos, como los elaborados por los jesuitas que mostraron similar respeto por la medicina indígena. En el siglo XVII, el saber curativo andino siguió gozando de estima, hasta el punto que muchos académicos de la época se opusieron a la apertura de una carrera de medicina por considerar que "los indios curan mejor que los médicos", tal como lo afirmó el Dr. Monzo de Huerta en 1637 (Ugarte 1965: 9). 
En el siglo XVIII, la influencia de la Ilustración con su eurocentrismo generó una mayor indiferencia respecto al saber curativo andino en comparación con los siglos anteriores. No obstante, el nacionalismo ilustrado contribuyó a trabajos botánicos importantes, como los publicados por Unanue en el Mercurio Peruano. Estas publicaciones incluyeron el conocimiento andino sobre las propiedades curativas de las plantas peruanas, debido a que este saber ya formaba parte de la cultura común de la sociedad colonial, pero lo que no estuvo presente fue la terapéutica ni la diagnosis de la medicina tradicional.

Durante la primera centuria republicana (siglo XIX) hubo desinterés por la medicina indígena y la cultura andina en general. Es probable que el darwinismo social influyera mucho. Luego de la guerra con Chile, recién las élites políticas e intelectuales peruanas mostraron mayor interés por lo andino, básicamente por una razón de identidad nacional. Así, el indigenismo de inicios del siglo XX tuvo influencia en la medicina y dio lugar a trabajos importantes de recopilación del saber tradicional. Posiblemente, el texto paradigmático de este tiempo sea La medicina popular peruana de Hermilio Valdizán y Ángel Maldonado (1922), una importante recopilación en tres tomos del saber tradicional andino a inicios del siglo XX, cuyo objetivo principal es generar un mayor acercamiento cultural con los connacionales andinos ${ }^{14}$. La mirada no es la del siglo XVI o XVII, sino la del médico moderno que debe conocer las "creencias prejuiciosas" de sus pacientes para tener más posibilidades de convencimiento al aplicar la medicina occidental.

14 Ya en un texto anterior (Nuestra medicina popular de 1911) Valdizán había afirmado que su trabajo era una "voz de alerta del camarada que comprende los peligros que significa para el médico su total ignorancia de los prejuicios del público cuya salud le ha sido confiada” (p. 1). 
Esta mirada sobre la medicina tradicional se mantuvo vigente hasta fines del siglo XX, cuando la nueva perspectiva médica mundial comenzó a influir en nuestros médicos. Fue clave la realización de la Conferencia Internacional de Alma Ata de la OMS en 1978. En este encuentro médico mundial, la OMS consideró a la medicina tradicional como una valiosa aliada que puede contribuir a mejorar la salud de las personas de determinado contexto, como en el caso del tercer mundo. Esta nueva postura dio lugar a la fundación en el Perú del INMETRA (Instituto Nacional de Medicina Tradicional) en 1990, que mostró un renovado interés y respeto por la medicina tradicional peruana.

A pesar del trabajo del INMETRA - que llegó a publicar una serie de 24 Cuadernos sobre Medicina Tradicional-, de algunos antropólogos contemporáneos como Mario Polia o Hugo Delgado Súmar, o de la labor de un historiador con conocimientos antropológicos como Luis Millones, aún es bastante exiguo el conocimiento que tenemos sobre la medicina tradicional peruana. Pero es claro que existe un renovado interés por comprender el saber tradicional médico, no solo por razones culturales, sino por su valor tangible como medicina en sí.

Espero que gracias a este texto muchos jóvenes investigadores se animen a historiar más y mejor la medicina tradicional peruana, que es sumamente valiosa y que sigue teniendo vigencia en nuestra sociedad. Todavía falta una obra sintética que permita entender la evolución de la misma y confío en que esta investigación signifique un primer paso hacia ese objetivo.

Recibido: 30 de junio del 2015.

Aceptado: 06 de noviembre del 2015. 


\section{Bibliografia}

ACOSTA, Joseph de

2006 Historia natural y moral de las Indias (edición de Edmundo Gorman; 3ra ed.). México, D.F.: Fondo de Cultura Económica.

CABIESES, Fernando

1985

"Una joya bibliográfica". Prólogo a la edición facsimilar de la obra La medicina popular peruana de Valdizán y Maldonado. Lima: CISA.

1974 Dioses y enfermedades: la medicina en el antiguo Perú. Dos volúmenes. Lima: Artegraf.

CHIAPPE, Mario; Moisés LEMLIJ y Luis MILLONES

1985 Alucinógenos y shamanismo en el Perú contemporáneo Lima: El Virrey.

COBO, Bernabé

$1964 \quad$ Obras del P. Bernabé Cobo de la Compañia de Jesús (edición de la Biblioteca de Autores Españoles, BAE). Madrid: Ediciones Atlas.

CUETO, Marcos

1989 Excelencia cientifica en la periferia: actividades cientificas e investigación biomédica en el Perú 1890-1950. Lima: GRADE.

112 DELGADO SÚMAR, Hugo E.

1998 La medicina tradicional en Lima: Inmigrantes de segunda y tercera generación. Tesis (Magíster en Antropología). Lima: Pontificia Universidad Católica del Perú.

1989a La tuna en la medicina tradicional. Ayacucho: Universidad Nacional de San Cristóbal de Huamanga, Facultad de Ciencias Sociales. (Cuadernos de Investigación, número 9). 
1989b Los curiosos en la medicina tradicional. Universidad $\mathrm{Na}$ cional de San Cristóbal de Huamanga, Facultad de Ciencias Sociales. (Cuadernos de Investigación, número 11 y 14).

1989c Rituales y procedimientos curativos en Ayacucho. Ayacucho: Universidad Nacional de San Cristóbal de Huamanga, Facultad de Ciencias Sociales. (Cuadernos de Investigación, número 7 ).

1988 Medicina tradicional en Ayacucho. Testimonio. Ayacucho, Universidad Nacional de San Cristóbal de Huamanga.

s.f. Saludy enfermedad en el mundo andino. Lima: Ministerio de Salud, Instituto Nacional de Medicina Tradicional.

FERNÁNDEZ IBARGUEN, Manuel

2000 "Medicina tradicional". En SALAVERRY, Oswaldo (editor) y Gustavo DELGADO MATALLANA (compilador). Historia de la medicina peruana del siglo XX. Lima: Universidad Nacional Mayor de San Marcos.

FUJII, Tatsuhiko, Luis MILLONES y Hiroyasu TOMOEDA (editores)

2004 Entre Dios y el diablo: magia y poder en la costa norte del Perú. Lima: Instituto Francés de Estudios Andinos, Pontificia Universidad Católica del Perú.

GARCÍA CÁCERES, Uriel

2005 "Hermilio Valdizán (1885-1929) y su obra Historia de la medicina peruana". En VALDIZÁN, Hermilio. Historia de la medicina peruana. Tercera edición. Lima: Instituto Nacional de Cultura.

GRIFFITHS, Nicholas

1998 La cruzy la serpiente: la represión y el resurgimiento religioso en el Perú colonial. Lima: Fondo Editorial PUCP. 
HUERTA, Elmer

2015 "Un antibiótico medieval barre con moderna superbacteria”. El Comercio, 6 de abril, p. A14.

LEMLIJ, Moisés y Luis MILLONES (editores)

1994 En el nombre del Señor: shamanes, demonios y curanderos del norte del Perú Lima: Biblioteca Peruana de Psicoanálisis.

MATEOS, Francisco

1964

"Introducción". En COBO, Bernabé. Obras del P. Bernabé Cobo de la Compañía de Jesús (edición de la Biblioteca de Autores Españoles, BAE). Madrid: Ediciones Atlas, tomo 1. p. XX.

MERCURIO PERUANO

[1791-1795] Periódico bisemanal. Lima.

MILLONES, Luis

2002 Las confesiones de don Juan Varquez. [1710]. Lima: Instituto Francés de Estudios Andinos, Fondo Editorial PUCP.

\section{MINISTERIO DE SALUD}

1990 Ley de Organización y Funciones del Ministerio de Salud. Lima, 16 de abril de 1990. Decreto Legislativo $N^{\circ}$ 584. <ftp://ftp.minsa.gob.pe/intranet/leyes/ DL-584_LOF-MINSA.pdf>

ORGANIZACIÓN MUNDIAL DE LA SALUD (OMS)

2005 Estrategia de la OMS sobre medicina tradicional 20022005. Ginebra: OMS.

POLIA, Mario

2001 La sangre del cóndor: chamanes de los Andes. Lima: Fondo Editorial del Congreso del Perú.

POLO DE ONDEGARDO, Juan

1616 [1585] Los errores y supersticiones de los indios, sacadas del tratado y averiguación que hizo el Licenciado Polo. En ROME- 
RO, Carlos y Horacio URTEAGA. Lima; Imprenta y Librería Sanmartí. Colección de Libros y Documentos referentes a la Historia del Perú, volumen III.

POMA DE AYALA, Guamán

[1615] El primer nueva corónica y buen gobierno (1615-1616). [Manuscrito original de la Biblioteca Real de Dinamarca]. København, Det Kongelige Bibliotek, GKS $22324^{\circ}$. <http://www.kb.dk/permalink/2006/ poma/840/es/text/>

RAIMONDI, Antonio

1940 [1874] El Perú. Parte preliminar. Lima: Escuela Tipográfica Salesiana.

SALAVERRY, Oswaldo (editor) y Gustavo DELGADO MATALLANA (compilador)

$2000 \quad$ Historia de la medicina peruana del siglo XX. Dos volúmenes. Lima: Universidad Nacional Mayor de San Marcos.

TAYLOR, Gerald

2003 Camac, camay y camasca. Lima: CBC, Instituto Francés de Estudios Andinos.

UGARTE, Luis A.

1965 Elpensamiento médico en el Perú virreinal. Lima: Universidad Nacional Mayor de San Marcos.

VALDIZÁN, Hermilio

2005 [1939] Historia de la medicina peruana. Tercera edición. Lima: Instituto Nacional de Cultura.

1911a La Facultad de Medicina de Lima, 1811-1911. Lima: Imprenta Sanmartí y Ca.

1911b Nuestra medicina popular. Apuntes para su historia. Lima: Sanmartí y Ca. 
Apuntes para la elaboración de una historia de la medicina tradicional andina

VALDIZÁN, Hermilio y Ángel MALDONADO

1922 La medicina popular peruana. Lima: Imprenta Torres Aguirre.

VALEGA, Juan Francisco

1935 Mis recuerdos de Hermilio Valdizán. Lima.

VALLE Y CAVIEDES, Juan del

1990 Obra completa. Lima: Banco de Crédito del Perú. Biblioteca Clásicos del Perú, número 5.

VARESE, Stefano

1965 Antonio Raimondi. Lima: Editorial Litográfica "La Confianza". 